\title{
Phase-locked stationary soliton states in birefringent nonlinear optical fibers
}

\author{
N. N. Akhmediev and A. V. Buryak \\ Optical Sciences Center, Australian National University, Canberra, A.C.T. 0200, Australia
}

J. M. Soto-Crespo

Instituto de Óptica, Consejo Superior de Ivestigaciones Cientificas, Serrano 121, 28006 Madrid, Spain

\author{
D. R. Andersen
}

Department of Electrical and Computer Engineering, University of Iowa, Iowa City, Iowa 52242

Received January 25, 1994; revised manuscript received July 1, 1994

\begin{abstract}
We consider stationary soliton states in a birefringent optical fiber with two components locked in phase. Two values of the phase difference between the two components of the soliton states are studied: 0 and $\pi / 2$. These cases allow us to find composite soliton states in a simple way. The bifurcation diagrams for the coupled soliton states in these two cases are constructed. The stability of these soliton states is examined also.
\end{abstract}

Propagation of soliton pulses in birefringent nonlinear fibers has been studied intensively in recent years. ${ }^{1-11}$ It was shown by Menyuk ${ }^{7}$ that two extreme cases are of importance: the approximation of high birefringence and the approximation of low birefringence. The approximation of high birefringence was considered in detail in Ref. 7. In this approximation the components of the field have different phase velocities and different group velocities. Owing to the nonlinearity, the pulses in these two components can capture each other, but their frequencies (and phases) become different. The approximation of low birefringence, where the difference in phase velocities of two components is taken into account but the difference in group velocities is ignored, was considered numerically by Blow et al. ${ }^{2}$ In particular, polarization instabilities were found in Ref. 2. Polarization instabilities were studied in more detail by Wright et al. ${ }^{8}$ In the present paper we concentrate on the approximation of low birefringence. Specifically, we find the solutions that are locked in phase, and we study the stability properties of these solutions.

It has been shown by Evangelides et al. ${ }^{11}$ through extensive numerical simulations, that the same state of polarization applies to a soliton as a whole. This means that the phase difference between the two components of the solitonlike pulses in a birefringent optical fiber is fixed across the pulse. If the components are locked in phase, then the state of polarization can be fixed along the fiber as well. These types of solution are the subject of investigation in this paper.

The coupled nonlinear Schrödinger equations (NLS's) describing pulse propagation in a linearly birefringent lossless fiber are ar $^{11}$

$$
\begin{array}{r}
i\left(\frac{\partial U}{\partial \xi}+\delta \frac{\partial U}{\partial \tau}\right)+\frac{1}{2} \frac{\partial^{2} U}{\partial \tau^{2}}+\beta U+\left(|U|^{2}+A|V|^{2}\right) U \\
+(1-A) V^{2} U^{*}=0
\end{array}
$$

$$
\begin{aligned}
i\left(\frac{\partial V}{\partial \xi}-\delta \frac{\partial V}{\partial \tau}\right)+\frac{1}{2} \frac{\partial^{2} V}{\partial \tau^{2}}-\beta V & +\left(A|U|^{2}+|V|^{2}\right) V \\
& +(1-A) V^{2} V^{*}=0
\end{aligned}
$$

where $U$ and $V$ are slowly varying components of the field on the slow and the fast axes, respectively, $2 \beta$ is the wave-number difference ( $\pi / \beta$ is the beat length), $2 \delta$ is the corresponding inverse group-velocity difference, and $A$ is the cross-phase-modulation coefficient. All variables and parameters in Eqs. (1) are normalized. $A$ is equal to $2 / 3$ in the case of a linearly birefringent fiber, but it can be different in more complicated cases. ${ }^{7}$

Equations (1) are complex and are difficult to solve in general. They are usually simplified. One way to simplify them is to represent the field components in the form

$$
\begin{aligned}
& U(\xi, \tau)=u(\tau, \xi) \exp (i \beta \xi), \\
& V(\xi, \tau)=v(\tau, \xi) \exp (-i \beta \xi)
\end{aligned}
$$

Then Eqs. (1) can be written in the form

$$
\begin{aligned}
& i\left(\frac{\partial u}{\partial \xi}+\delta \frac{\partial u}{\partial \tau}\right)+ \frac{1}{2} \frac{\partial^{2} u}{\partial \tau^{2}}+\left(|u|^{2}+A|v|^{2}\right) u \\
&+(1-A) v^{2} u^{*} \exp (-i 4 \beta \xi)=0 \\
& i\left(\frac{\partial v}{\partial \xi}+\delta \frac{\partial v}{\partial \tau}\right)+\frac{1}{2} \frac{\partial^{2} v}{\partial \tau^{2}}+\left(A|u|^{2}+|v|^{2}\right) v \\
&+(1-A) u^{2} v^{*} \exp (i 4 \beta \xi)=0
\end{aligned}
$$

with rapid phase variations along $\xi$ in the last terms. The last terms are usually neglected, ${ }^{7}$ because, when averaged over $\xi$, they equal zero. Then the factor in the cross-phase-modulation term $(A)$ is exactly $2 / 3$ of the factor in the self-phase-modulation term. 
However, this approximation can be made if $\beta$ is high and the two components are propagating with different phase velocities. ${ }^{7}$ Then birefringence manifests itself only in the different group velocities of the two components. Because of the nonlinearity, pulses consisting of two components can still move with the same group velocity, but then the two components must have different frequencies. ${ }^{7}$ Consequently the phases of the two components rotate relative to each other. This can also be seen clearly from Eqs. (3): without the third nonlinear coupling terms [proportional to $(1-A)$ ], each component of the field can be multiplied by an arbitrary phase factor that does not depend on the phase of the other component.

In the case of low birefringence the difference in group velocities can be ignored. The effect of birefringence is taken into account in equations as the difference in phase velocities. Nonlinearity does not shift the frequencies of the two components, but it can change the phase velocities. In this case the third nonlinear coupling term [proportional to $(1-A)$ ] becomes important. Now any changes in the phase of one component must be related to phase changes of another component. As a result the two components of the field can be locked in phase.

In fact, when representing the fields in form (2), one assumes that the two field components have different phase velocities along the fiber, as in the linear limit. In the nonlinear case two coupled components of the field can be locked in phase as a result of the nonlinear changes in phase velocities, and thus the last terms in Eqs. (1) become important and cannot be neglected or averaged. Because of the phase locking, instead of substitution (2), we separate out the common fast oscillatory part of the functions $U$ and $V$ in the form

$$
\begin{aligned}
& U(\xi, \tau)=u(\tau, q) \exp (i q \xi) \\
& V(\xi, \tau)=v(\tau, q) \exp (i q \xi+i \phi),
\end{aligned}
$$

where $u$ and $v$ are soliton envelopes, $q$ is the common spatial frequency, and $\phi$ is the phase difference between the $U$ and the $V$ functions.

We consider here only stationary solutions in $\xi$, so that the functions $u$ and $v$ do not depend on $\xi$. However, these functions are complex and can have complicated forms in $\tau$. The shape of solitons now depends on $q$, which can be considered an independent parameter of the solitonstate family of solutions. As soon as two components $U$ and $V$ are locked in phase, the relative phase $\phi$ between them becomes important. It can be considered a second independent parameter of soliton-state families.

Since the functions $u$ and $v$ are complex, we have to solve four equations in four real unknown functions. However, there are two cases for which this set of equations can be reduced to two real equations. In this paper we consider only these two simplest cases. Each of them has no group-velocity difference $(\delta=0)$.

1. Both functions $U$ and $V$ have the same phase $(\phi=0)$. Without loss of generality, we can consider $u$ and $v$ purely real:

$$
U(\xi, \tau)=u(\tau, q) \exp (i q \xi), \quad V(\xi, \tau)=v(\tau, q) \exp (i q \xi) .
$$

2. The function $V$ is $\pi / 2$ out of phase with the function $U$ (i.e., $\phi=\pi / 2$ ). Thus we can consider $u$ real and the other envelope component purely imaginary. Transformations (4) then can be written as

$$
\begin{aligned}
& U(\xi, \tau)=u(\tau, q) \exp (i q \xi), \\
& V(\xi, \tau)=i v(\tau, q) \exp (i q \xi),
\end{aligned}
$$

where $v$ is again purely real.

Substituting Eqs. (5a) or (5b) into Eq. (1) and taking into account that $\delta=0$, we obtain the following set of equations:

$$
\begin{aligned}
& \frac{1}{2} \frac{\partial^{2} u}{\partial \tau^{2}}-(q-\beta) u+\left(u^{2}+\gamma v^{2}\right) u=0, \\
& \frac{1}{2} \frac{\partial^{2} v}{\partial \tau^{2}}-(q+\beta) v+\left(\gamma u^{2}+v^{2}\right) v=0,
\end{aligned}
$$

where $\gamma=A+(1-A)=1$ in case 1 and $\gamma=2 A-1=1 / 3$ in case 2 . We remind the reader that $u$ and $v$ in Eqs. (6) are real in both cases.

The difference between the two cases comes from the terms responsible for the cross-phase modulation. We use this name for the terms with the factor $\gamma$, although after the transformations that we have made above they are not conventional cross-phase-modulation terms. If we choose the relative phase between $U$ and the $V$ functions to be neither 0 nor $\pi / 2$, then the functions $u$ and $v$ are complex and have a complicated dependence on $\tau$. We leave the consideration of this general problem for the future.

With the substitution

$$
x=\frac{\sqrt{1+\gamma}}{2}(u+v), \quad y=\frac{\sqrt{1+\gamma}}{2}(u-v),
$$

Eqs. (6) with $\gamma=1 / 3$ can be further transformed to describe propagation of two circularly polarized waves with amplitudes $x$ and $y$ :

$$
\begin{aligned}
& \frac{1}{2} \frac{\partial^{2} x}{\partial \tau^{2}}-q x+\beta y+\left(x^{2}+\frac{3-\gamma}{1+\gamma} y^{2}\right) x=0, \\
& \frac{1}{2} \frac{\partial^{2} y}{\partial \tau^{2}}-q y+\beta x+\left(\frac{3-\gamma}{1+\gamma} x^{2}+y^{2}\right) y=0 .
\end{aligned}
$$

This transformation is the particular case of general transformations that were performed previously in Ref. 2 . We can see that the cross-phase-modulation coefficient in Eqs. (8) is equal to $\gamma^{\prime}=(3-\gamma) /(1+\gamma)$ (so $\gamma^{\prime}=2$ for $\gamma=1 / 3$ ). However, in contrast to Eqs. (6), Eqs. (8) have linear terms that are responsible for energy exchange between the modes. This transformation shows that Eqs. (6) with $\gamma=1 / 3$ and Eqs. (8) with $\gamma^{\prime}=2$ have physically equivalent solutions.

Now we concentrate on the solutions of Eqs. (6). We consider two limiting cases, $\gamma=1$ and $\gamma=1 / 3$. The set of Eqs. (6) with $\gamma=1$ is integrable. Its lowest-order soliton solutions follow:

Solitons polarized along the $u$ axis,

$$
u=\sqrt{2(q-\beta)} \operatorname{sech}[\sqrt{2(q-\beta)} \tau], \quad v=0 ;
$$

Solitons polarized along the $v$ axis, 


$$
u=0, \quad v=\sqrt{2(q+\beta)} \operatorname{sech}[\sqrt{2(q+\beta)} \tau] .
$$

For these two solutions the phases of the two components are independent. The phases of the two components are locked in the following composite (nonpolarized) solitonstate solutions ${ }^{12,13}$ :
Eqs. (9) and (10) ( $\mathrm{F}_{u}$ - and $\mathrm{F}_{v}$-type branches). More complicated solutions of Eqs. (6) cannot be found analytically, but it is possible to find them numerically. ${ }^{12,14}$ Analytically we can find only the points of bifurcation at which these states split off from the polarized ones.

It can be shown that there is no bifurcation from the polarized states, Eqs. (9), in the range $0<\gamma<1$. To

$$
\begin{aligned}
& u=-\frac{\lambda_{2} \sqrt{\lambda_{1}^{2}-\lambda_{2}^{2}} \sinh \left[\lambda_{1}\left(\tau-\tau_{1}\right)\right]}{\lambda_{1} \cosh \left[\lambda_{1}\left(\tau-\tau_{1}\right)\right] \cosh \left[\lambda_{2}\left(\tau-\tau_{2}\right)\right]-\lambda_{2} \sinh \left[\lambda_{1}\left(\tau-\tau_{1}\right)\right] \sinh \left[\lambda_{2}\left(\tau-\tau_{2}\right)\right]}, \\
& v=\frac{\lambda_{1} \sqrt{\lambda_{1}^{2}-\lambda_{2}^{2}} \cosh \left[\lambda_{2}\left(\tau-\tau_{2}\right)\right]}{\lambda_{1} \cosh \left[\lambda_{1}\left(\tau-\tau_{1}\right)\right] \cosh \left[\lambda_{2}\left(\tau-\tau_{2}\right)\right]-\lambda_{2} \sinh \left[\lambda_{1}\left(\tau-\tau_{1}\right)\right] \sinh \left[\lambda_{2}\left(\tau-\tau_{2}\right)\right]},
\end{aligned}
$$

where $\lambda_{1}=\sqrt{2(q+\beta)}, \quad \lambda_{2}=\sqrt{2(q-\beta)}$, and $\tau_{1}$ and $\tau_{2}$ are arbitrary real constants. The solution, Eqs. (11), at each fixed $q$ is still a two-parameter family of solutions (effectively these two parameters can be reduced to one parameter, $\Delta \tau=\tau_{1}-\tau_{2}$, which corresponds to the distance between the two solitons involved in the nonlinear superposition). The particular case corresponding to Eqs. (11) at $\Delta \tau=\left[\left(\lambda_{2}-\lambda_{1}\right) /\left(2 \lambda_{2} \lambda_{1}\right)\right] \ln \left[\left(\lambda_{2}+\lambda_{1}\right) /\left(\lambda_{2}-\lambda_{1}\right)\right]$ was obtained in Ref. 5 . In the limit $q=\beta$, the solution, Eqs. (11), converges to the polarized solution, Eqs. (10).

A convenient way to consider the soliton states is to represent them on the energy-versus-spatial-frequency diagram. ${ }^{14}$ The total energy carried by the coupled soliton states is defined by

$$
\begin{aligned}
Q & =\int_{-\infty}^{\infty}\left(|U|^{2}+|V|^{2}\right) \mathrm{d} \tau=\int_{-\infty}^{\infty}\left(u^{2}+v^{2}\right) \mathrm{d} \tau \\
& =\frac{2}{1+\gamma} \int_{-\infty}^{\infty}\left(x^{2}+y^{2}\right) \mathrm{d} \tau
\end{aligned}
$$

The energies of two polarized solitons, Eqs. (9) and (10), are given by $Q=2 \sqrt{2(q-\beta)}$ and $Q=2 \sqrt{2(q+\beta)}$, respectively. The energy of the soliton state, Eqs. (11), is given by

$$
Q=2 \sqrt{2(q-\beta)}+2 \sqrt{2(q+\beta)} .
$$

The curves $Q(q)$, with $Q$ and $q$ normalized by the parameter $\beta$, for three different types of soliton state are shown in Fig. 1(a). The energy dispersion curves for solutions (9) and (10) have square-root dependencies on $q$ and are shifted with respect to each other because of their different phase velocities. We denote these curves $\mathrm{F}_{u}$ and $\mathrm{F}_{v}$ respectively. The soliton states, Eqs. (11), bifurcate from curve $\mathrm{F}_{v}$ at the point $q=\beta$. This bifurcation point is denoted point $\mathrm{H}$. We call this new branch of solutions the C-type soliton states. Each point of this branch corresponds to an infinite number of different soliton states [different $\Delta \tau$ 's in Eqs. (11)]. This is the consequence of the integrability of the set of Eqs. (6) with $\gamma=1$. Examples of the envelope functions $u$ and $v$ at some point of the branch $\mathrm{C}$ for two different values of $\Delta \tau$ are given in Fig. 2.

The polarized solitons of the set of Eqs. (6) with $\gamma=1 / 3$ are the same as for the set of Eqs. (6) with $\gamma=1$, i.e., study the bifurcation from the states of Eqs. (10), we perturb these solutions in a specific way:

$$
u=\epsilon G, \quad v=\alpha \operatorname{sech}(\alpha t)+\epsilon^{2} F,
$$

where $\alpha=[2(q+\beta)]^{1 / 2}$ and $\epsilon$ is a small parameter. Substituting Eqs. (14) into Eqs. (6) and linearizing with respect to the small parameter $\epsilon$, we find
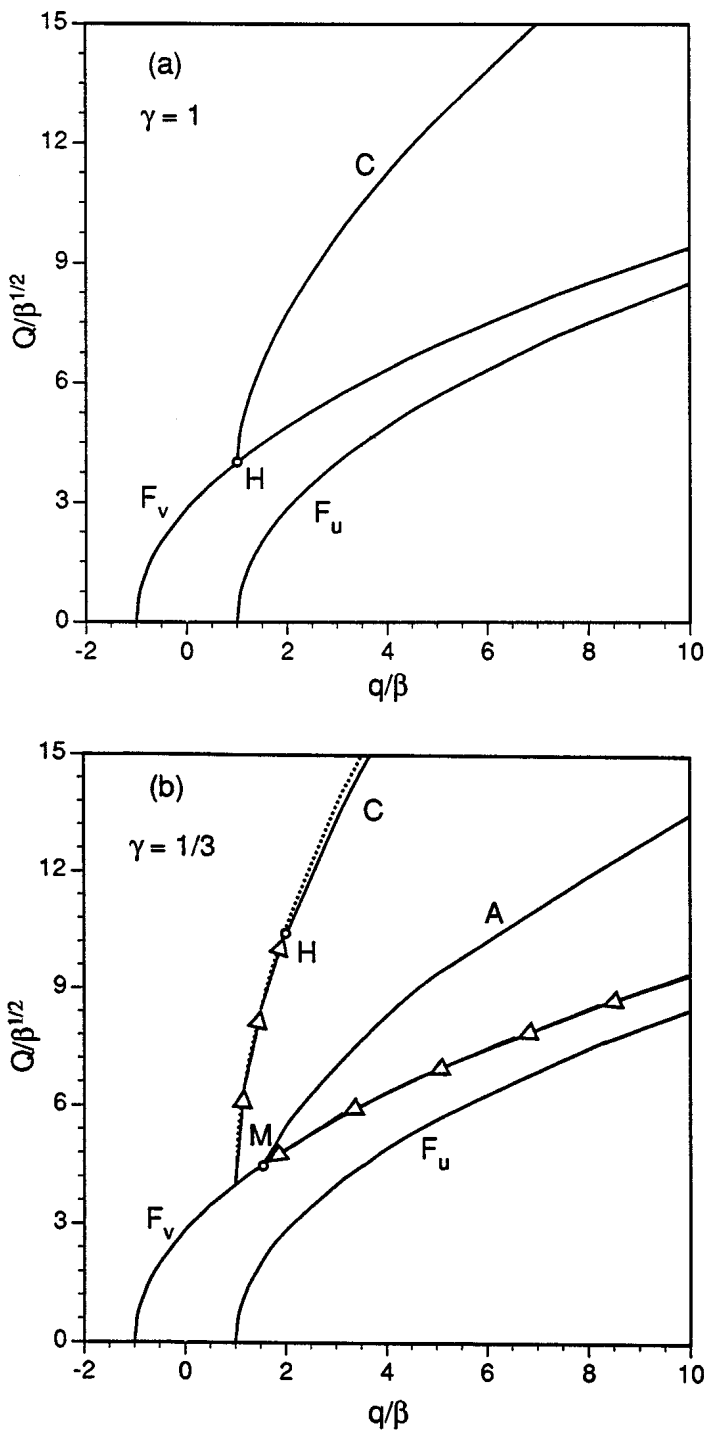

Fig. 1. Bifurcation diagram for polarized and composite soliton states in a birefringent optical fiber: (a) $\gamma=1$, (b) $\gamma=1 / 3$. 

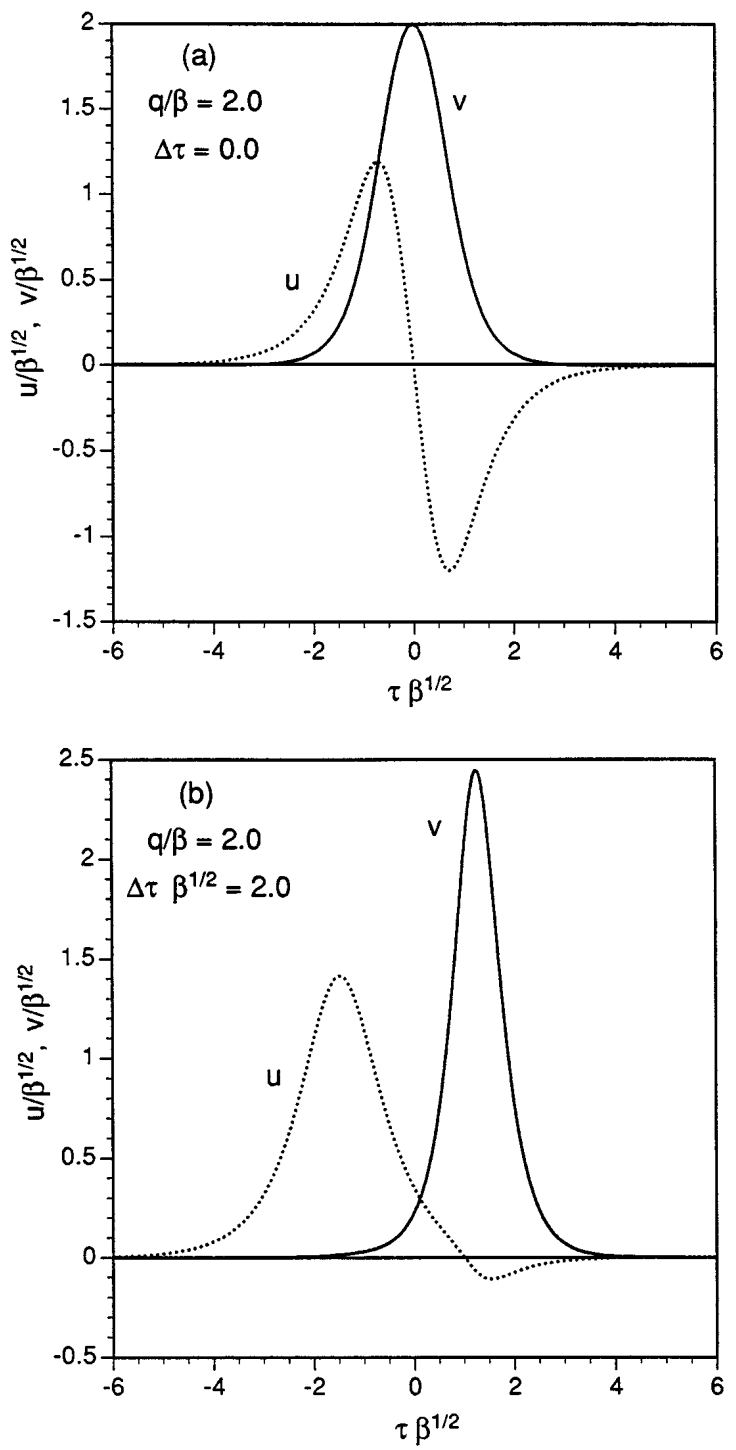

Fig. 2. Examples of functions $u$ and $v$ for the C-branch soliton states at $q / \beta=2.0$ : (a) $\Delta \tau=0$, (b) $\Delta \tau \sqrt{\beta}=2.0$.

$$
\ddot{G}-\mu^{2} \alpha^{2} G+\frac{2 \gamma \alpha^{2}}{\cosh ^{2} \alpha t} G=0,
$$

where $\mu=[(q-\beta) /(q+\beta)]^{1 / 2}$. When $\gamma$ is in the interval $0<\gamma<1$, then only the lowest-order even bounded solution exists when the condition

$$
2 \gamma=\mu(\mu+1)
$$

is fulfilled. The soliton states, Eqs. (10), exist at $q>-\beta$. In the case $\gamma=1 / 3$ we then have $q / \beta=\left(1+\mu^{2}\right) /(1-$ $\left.\mu^{2}\right) \approx 1.529$. We call the composite states, which split off from the $\mathrm{F}_{v}$-type soliton states at this point, A-type soliton states. The set of curves for different types of soliton state at $\gamma=1 / 3$ is shown in Fig. 1(b). The arrows in this figure show the direction in which the bifurcation points shift as the value of $\gamma$ in Eqs. (6) changes from 1 to $1 / 3$. At $\gamma=1$ curve $\mathrm{C}$ bifurcates from the $\mathrm{F}_{v}$-type polarized states at the point $q / \beta=1$ (point $H$ ). The other bifurcation point is formally located at $q / \beta=+\infty$. While $\gamma$ changes from 1 to $1 / 3$, point $H$ moves along the curve $Q=4[2(q-\beta)]^{1 / 2}+2[2(q+\beta)]^{1 / 2}$ from $q / \beta=1$ to $q / \beta \approx 1.834$, and point $\mathrm{M}$ moves along the $\mathrm{F}_{v}$-type polarized branch from $q / \beta=+\infty$ to $q / \beta \approx 1.529$. At $\gamma \neq 1$ the integrability is lost. The consequence is that any point of curve $\mathrm{C}$ in Fig. 1(b) corresponds to a single soliton state with fixed $\Delta \tau=0$.

An example of the envelope functions $u$ and $v$ at some point of branch $\mathrm{A}$ (which bifurcates at point $\mathrm{M}$ ) calculated numerically, is given in Fig. 3. The smaller component ( $u$ in Fig. 3 ) is usually considered a shadow. ${ }^{7}$ In principle it is an essential part of the composite soliton state, rather than just a shadow of the larger component. The whole pulse can be considered to be elliptically polarized with its larger axis of polarization along the $v$ axis, because component $v$ has a bigger amplitude, and the phase difference between the two components is $\pi / 2$.

The stability of soliton states in this kind of problem was considered, for example, in Refs. 8 and 15 . We studied the stability of the soliton states presented above by the linearization method. ${ }^{16-19}$ Although the case of the single NLS was studied in Refs. 16-19, the linearization method can be extended to cover the coupled NLS case as well. The numerical technique for calculating the eigenfunctions of the perturbation and the instability growth rates for NLS-type equations with a linearized parabolic equation used for the perturbation is presented in Ref. 20. The results are shown in Fig. 4. The instability growth rate for the fast mode is shown in Fig. 4 by the solid curves. There are two intervals of $q / \beta$ for which the fast mode is unstable. The region of instability to the right of the bifurcation point at $q / \beta>1.53$ relates to the appearance of the A-type states in this region and can be called the symmetry-breaking instability region. In the region to the left of $q / \beta>1.475$ the instability is related to the radiation of small-amplitude waves by the soliton.

We found that the A-type soliton states are unstable in the whole region of the parameter $q / \beta$ where they exist. Their instability growth rate is shown by the lower dashed curve in Fig. 4. The growth rate is lower than the growth rate for branch $\mathrm{F}_{v}$ at the same value of $q / \beta$. This instability is related to the radiation of small-amplitude waves from the A-type soliton states. The perspective

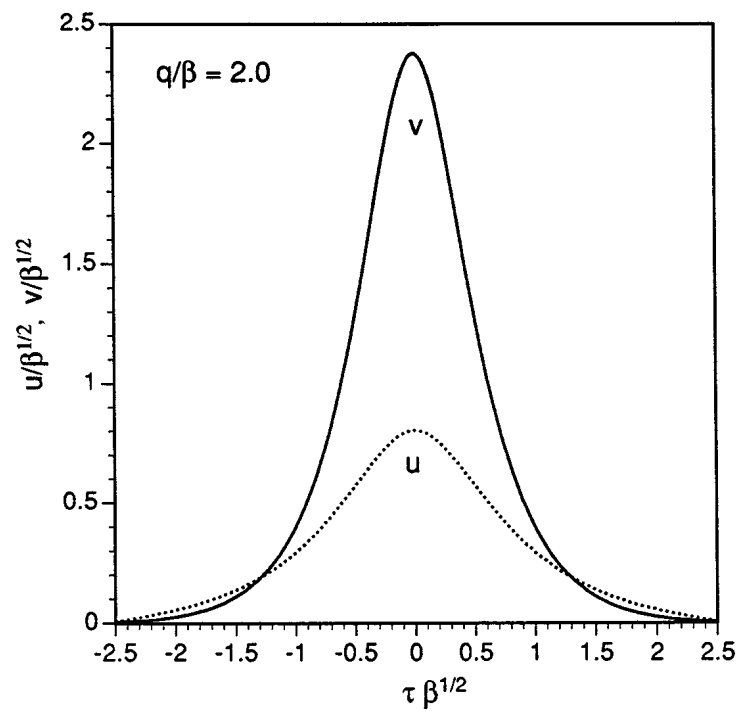

Fig. 3. Example of functions $u$ and $v$ for the A-branch soliton states at $q / \beta=2.0$. 


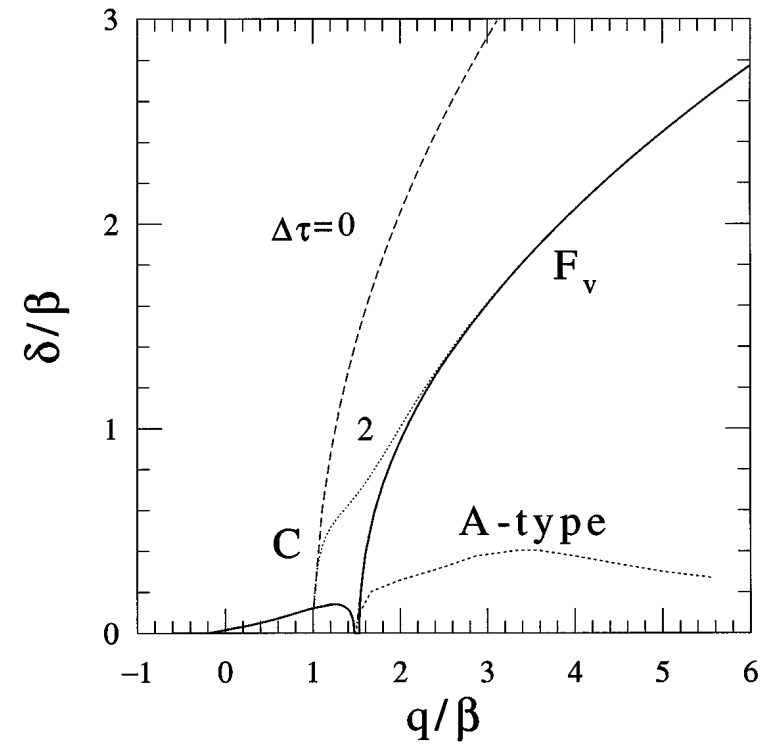

Fig. 4. Instability growth rates for the fast soliton (solid curves), for the A-type soliton states (lower dashed curve), and for the C-type soliton states at $\Delta \tau=0$ (leftmost dashed curve) at $\Delta \tau=2$ (dotted curve). The curve for $\Delta \tau=6$ practically coincides with the curve for the fast soliton.

plot of the evolution of the A-type states is shown in Fig. 5 . The figure shows that for small perturbations the A-type soliton state can propagate long distances without visible changes. A propagation distance larger than $\xi=300$ is necessary to let us appreciate a significant modification of the initial field profile. Then the pulse starts to oscillate around some stationary state. These oscillations are accompanied by the radiation of small-amplitude waves.

The C-type soliton states can be considered a combination of two solitons polarized along the fast and the slow axes. Therefore C-type states can be unstable, and the result of such instability will be disintegration of the soliton's constituents. We have observed this unstable behavior in our numerical solutions of Eqs. (1) for such input fields. Figure 6 shows the evolution of the C-type state corresponding to $q=1, \beta=0.01$, and $\Delta \tau=0$. Below a certain value of $\xi$ the soliton state remains stationary, as it corresponds to a stationary solution of Eqs. (1). Starting from some value of $\xi$ (in this particular case $\xi \approx 25$ ), which depends on the initial perturbation, the pulse splits into two pulses, which separate on propagation and whose amplitudes oscillate periodically. These two pulses are perturbed A-type states rather than linearly polarized solitons.

Figure 7 represents the real part versus the imaginary part of the field amplitudes at the center of the left and the right pulses. The variable $\xi$ in this figure changes from 150 to 300. L's in the figure indicate the trajectory for the left pulse, R's indicate the trajectories for the right pulse, and $U$ and $V$ indicate the slow and the fast components, respectively. Trajectories are plotted in Fig. 7 with additional phase factors $\exp (0.498 \xi)$, for the left pulse and $\exp (1.411 \xi)$ for the right pulse to prevent their rotation around the origin. The arrows point to the center of those four quasi-elliptical trajectories. The solid arrows are for the left pulse and the dashed arrows for the right pulse. We can see that the angle formed between the solid (or dashed) arrows is constant and $\approx 90^{\circ}$. These four trajectories show that a periodic motion around two different A-type states with $q=0.498$ and $q=1.411(\beta=0.01)$ is taking place. Although the A-type states are unstable, their growth rates at high values of $q / b$ are negligible. The trajectories for the left
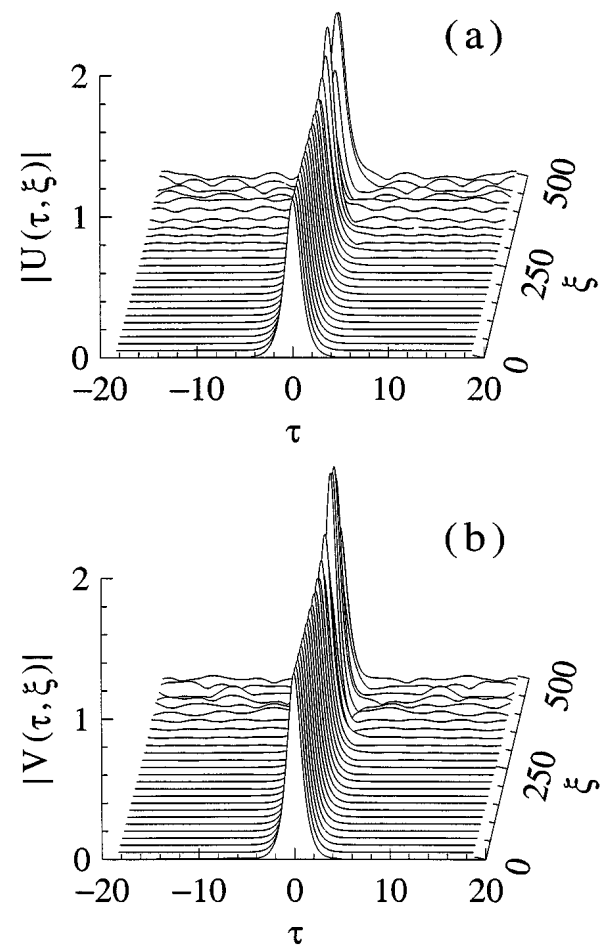

Fig. 5. Perspective plot for propagation of the A-type soliton states with a small initial perturbation. Parameters of the simulation are $A=2 / 3$ and $q / \beta=10$.
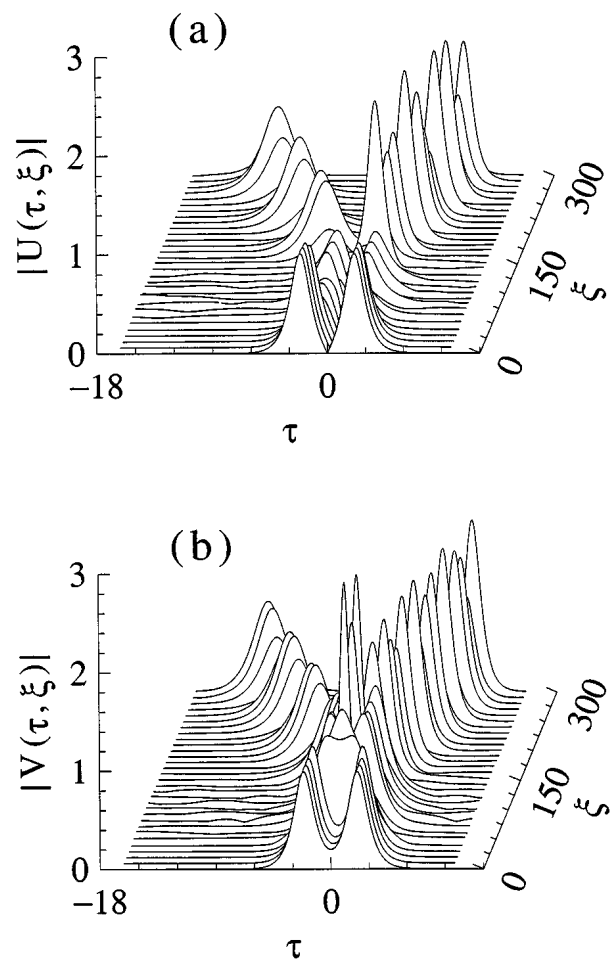

Fig. 6. Perspective plot for propagation of the C-type soliton states with a small initial perturbation. Parameters of the simulation are $A=2 / 3, \Delta \tau=0$, and $q / \beta=100$. 


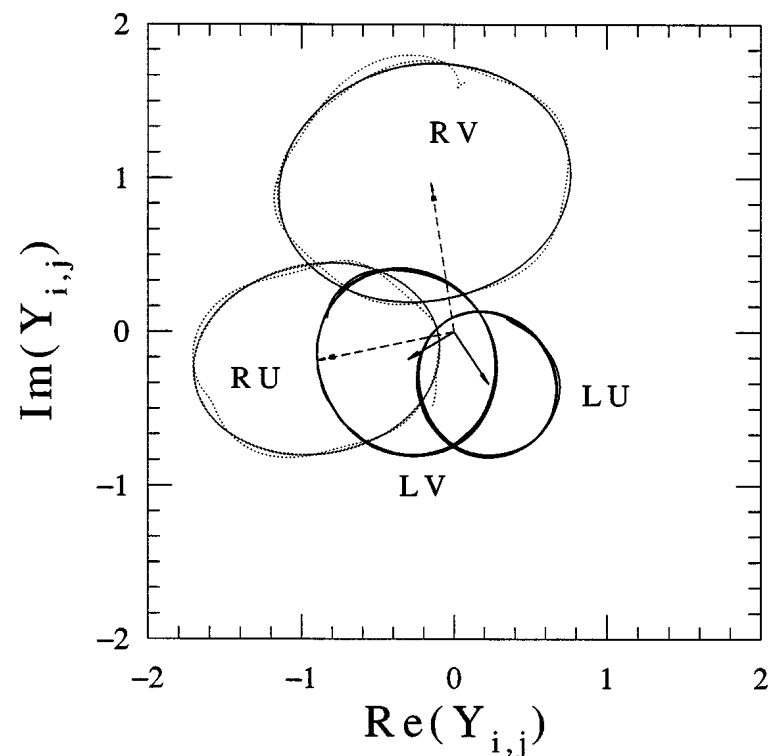

Fig. 7. Trajectories in the complex plane of variables $U$ and $V$ of the right (R) and the left (L) pulses of Fig. 6 after the C-type state splits into two pulses. Variables $Y_{i, j}$ denote $U$ or $V$ for the right or left pulses at the point of maximum amplitude.

pulse (solid curves) consist of three loops that are superimposed on one another with high accuracy. For the right pulse there are six periods of oscillation, and although the curve that represents them is dotted, the superposition is so good that it appears to be continuous.

This type of instability takes place in the whole region of $q / \beta$ where C-type soliton states exist. The instability growth rates for these solutions are shown in Fig. 4 . The leftmost dashed curve is the instability growth rate of the C-type solutions for $\Delta \tau=0$. For $\Delta \tau=6$ these solutions correspond practically to two temporarily separated linearly polarized pulses along the fast and the slow axes. As a result the growth rates coincide with those for the fast solitons of the same parameters $(q, \beta)$. In the intermediate case, $\Delta \tau=2$ (dotted curve), the growth rate follows the dashed curve at small $q / \beta$ (where $\Delta \tau$ is large relative to the width of the pulse).

Our simulations show that, generally speaking, the combined states of two solitons in the birefringent fiber are unstable even though they are stationary solutions of initial equations (1). This is in agreement with the numerical simulations of Ref. 2; Blow et al. noticed that any pulse with an energy higher than the energy of a single soliton breaks up into two or more pulses. Moreover, our numerical simulations show that input pulses of the composite shape can be split into two solitons that are A-type elliptically polarized solitons rather than linearly polarized ones. After some distance they can transform into slow, linearly polarized solitons, but this requires long propagation distances. On the other hand, A-type elliptically polarized pulses can be excited directly in a polarization-preserving fiber by a single solitonlike pulse if the input pulse has elliptic polarization, with the main axis of the ellipse being the fast principal axis of birefringence.

In conclusion, we have considered stationary soliton states in nonlinear birefringent fibers with two components locked in phase, investigated the influence of the phase difference between these two components on the structure of soliton states, and constructed the bifurcation diagrams for two simple cases. The stability of these soliton states is also studied numerically.

\section{ACKNOWLEDGMENTS}

The work of J. M. Soto-Crespo was supported by the Comisión Interministerial de Ciencia y Technología under contract TIC91-0361. The work of N. N. Akhmediev and A. V. Buryak is supported by the Australian Photonics Cooperative Research Centre. The authors are grateful to A. W. Snyder for useful discussions and to A. Ankiewicz for a critical reading of the manuscript.

\section{REFERENCES}

1. H. G. Winful, Appl. Phys. Lett. 47, 213 (1985).

2. K. J. Blow, N. J. Doran, and D. Wood, Opt. Lett. 12, 202 (1987).

3. C. R. Menyuk, Opt. Lett. 12, 614 (1987).

4. S. Wabnitz, Phys. Rev. A 38, 2018 (1988).

5. D. N. Christodoulides and R. I. Joseph, Opt. Lett. 13, 53 (1988).

6. S. Trillo, S. Wabnitz, E. M. Wright, and G. I. Stegeman. Opt. Lett. 13, 672 (1988).

7. C. R. Menyuk, IEEE J. Quantum Electron. 25, 2674 (1989)

8. E. M. Wright, G. I. Stegeman, and S. Wabnitz, Phys. Rev. A 40, 4455 (1989).

9. M. Haelterman and A. P. Sheppard, Electron. Lett. 29, 1176 (1993).

10. C. J. Chen, P. K. A. Wai, and C. R. Menyuk, Opt. Lett. 15, 477 (1990).

11. S. G. Evangelides, Jr., L. F. Mollenauer, J. P. Gordon, and N. S. Bergano, J. Lightwave Technol. 10, 28 (1992).

12. N. N. Akhmediev, V. M. Eleonskii, N. E. Kulagin, and L. P. Shil'nikov, Pis'ma Zh. Tekh. Fiz. 15, 19 (1989) [Sov. Tech. Phys. Lett. 15, 587 (1989)].

13. M. V. Tratnik and J. E. Sipe, Phys. Rev. A 38, 2011 (1988).

14. N. N. Akhmediev and A. Ankiewicz, Phys. Rev. Lett. 70, 2395 (1993).

15. J. M. Soto-Crespo and N. N. Akhmediev, Phys. Rev. E 48, 4710 (1993).

16. A. A. Kolokolov, J. Appl. Mech. Tech. Phys. (USSR) 11, 426 (1975).

17. C. K. R. T. Jones and J. V. Moloney, Phys. Lett. A 117,175 (1986).

18. D. J. Mitchell and A. W. Snyder, J. Opt. Soc. Am. B 10, 1572 (1993).

19. N. N. Akhmediev, "The problem of stability and excitation of nonlinear surface waves," in Nonlinear Surface Electromagnetic Phenomena, Vol. 29 of Modern Problems in Condensed Matter Sciences, H.-E. Ponath and G. I. Stegeman, eds. (North-Holland, Amsterdam, 1991), p. 289.

20. N. N. Akhmediev, V. I. Korneev, and Yu. V. Kuz'menko, Zh. Eksp. Teor. Fiz. 88, 107 (1985) [Sov. Phys. JETP 61, 62 (1985)] 